\title{
SACARIFICAÇÃO DE BAGAÇO DE CANA IN NATURA E PRÉ- TRATADO COM OZÔNIO POR ENZIMAS CELULOLÍTICAS PRODUZIDAS POR FUNGO TERMOFÍLICO EM CULTIVO SÓLIDO
}

\author{
P. A. CASCIATORI ${ }^{1}$, F. P. CASCIATORI ${ }^{2}$, J. C. THOMÉO $^{3}$ e R. da SILVA ${ }^{4}$ \\ ${ }^{1,4}$ Universidade Estadual Paulista, Instituto de Biociências, Letras e Ciências Exatas, Departamento \\ de Química e Ciências Ambientais \\ ${ }^{2,3}$ Universidade Estadual Paulista, Instituto de Biociências, Letras e Ciências Exatas, Departamento \\ de Engenharia e Tecnologia de Alimentos \\ E-mail para contato: pricasciatori@yahoo.com.br
}

\begin{abstract}
RESUMO - Enzimas celulolíticas são essenciais para a hidrólise de materiais lignocelulósicos visando à produção de etanol de segunda geração. A fermentação em estado sólido (FES) é um processo que proporciona altas concentrações de enzimas que podem ser aplicadas nessa hidrólise. O objetivo deste trabalho foi avaliar a eficiência do extrato produzido em FES pelo fungo termofílico Myceliophthora thermophila na hidrólise enzimática de bagaço de cana in natura e pré-tratado com ozônio. $\mathrm{O}$ planejamento experimental adotado para a sacarificação foi um planejamento de superfície de resposta central composto com 5 repetições do ponto central. Os fatores adotados foram volume de extrato enzimático aplicado e tempo de hidrólise. O teor de açúcares redutores no hidrolisado foi determinado pelo método DNS. Os estimadores estatísticos da hidrólise de bagaço in natura e pré-tratado não indicaram diferença significativa na quantidade de açúcares redutores totais liberados em função do volume de enzima e do tempo de hidrólise. Contudo, os melhores resultados foram obtidos empregando-se maiores volumes de enzima e tempo de hidrólise. Para os ensaios com o bagaço ozonolizado, notou-se um aumento de aproximadamente $80 \%$ da quantidade de açúcares redutores liberados em relação ao bagaço in natura. Assim, tem-se que a ozonólise é um pré-tratamento eficiente para aumentar a hidrólise enzimática, pois facilita a acessibilidade da celulase à celulose.
\end{abstract}

\section{INTRODUÇÃO}

Diante da perspectiva de esgotamento das jazidas de combustíveis fósseis em áreas de mais fácil acesso à exploração (que são as reservas de petróleo acima da camada de sal, denominadas póssal), as companhias petrolíferas buscam fontes de mais difícil acesso (a camada pré-sal). Tal prática resulta em elevação de custos e aumenta a possibilidade de desastres ambientais. Ademais, a preocupação mundial com o efeito estufa requer redução do consumo de combustíveis fósseis. Neste 
contexto, a produção de combustíveis limpos, como o etanol celulósico, em larga escala é estratégica e o mercado é promissor.

No Brasil, uma matéria-prima abundante para conversão a etanol de segunda geração é o bagaço da cana-de-açúcar, resíduo da produção do etanol de primeira geração. O bagaço de cana é predominantemente constituído por celulose, hemicelulose e lignina, sendo assim uma potencial fonte de açúcares fermentescíveis (Pandey et al., 2000). No entanto, a produção de álcool a partir do bagaço de cana tem como fator limitante sua recalcitrância. Sendo assim, pré-tratamentos químicos, físicos e/ou biológicos têm sido propostos, com o intuito de se facilitar o acesso das celulases aos seus substratos e obter maiores rendimentos na sacarificação enzimática.

A natureza da matéria presente no bagaço de cana evidencia a importância da etapa de prétratamento do material lignocelulósico, pois os componentes estruturais contidos na parede celular dos lignocelulósicos (celulose, hemicelulose e lignina) são fortemente associados, formando um complexo estável e recalcitrante. A parede celular da cana de açúcar é composta por uma malha de celulose contendo regiões amorfas e cristalinas e hemicelulose ligadas covalentemente à lignina, o que dificulta a ação de enzimas que poderiam hidrolisar esses compostos em açúcares fermentescíveis. A estrutura cristalina da celulose também representa um obstáculo extra à hidrólise enzimática. Somente com a remoção da lignina e a ruptura da celulose cristalina as enzimas teriam acesso aos polímeros de açúcar (Pinto, 2010).

O pré-tratamento tem como objetivo aumentar o tamanho dos poros e reduzir a cristalinidade da celulose, bem como reduzir a estabilidade das fibras, sendo utilizado com o intuito de reduzir a interação entre celulose, hemicelulose e lignina, solubilizando a hemicelulose e/ou a lignina, e tornando o substrato mais acessível às enzimas que convertem os carboidratos em açúcares fermentescíveis (Chang et al., 1998; Jorgensen et al., 2007). Assim, o pré-tratamento visa fundamentalmente o aumento da acessibilidade das enzimas à celulose.

A ozonólise é um tipo de processo oxidativo avançado, que pode ser utilizado no prétratamento químico da biomassa lignocelulósica e sua ação se deve à exposição do material a ozônio, um poderoso agente oxidante. O principal efeito deste pré-tratamento é a remoção da lignina e parte das hemiceluloses, favorecendo o aumento da digestibilidade enzimática do material pré-tratado (Quesada et al., 1999; Garcia-Cúbero et al., 2009). Algumas particularidades dos processos oxidativos envolvendo ozônio podem ser destacadas como: alta seletividade, fácil geração e ausência ou baixíssima concentração da formação de compostos tóxicos entre lignina e o oxidante. Além disso, o processo geralmente é conduzido à temperatura ambiente e não leva à formação de compostos inibidores de fermentação microbiana, como furfural e hidroximetilfurfural (Vidal e Molinier, 1988).

A despolimerização dos carboidratos a açúcares fermentescíveis pode ocorrer por duas vias: hidrólise enzimática ou química. A via química é eficiente e rápida, mas gera resíduos tóxicos que devem ser tratados, aumentando o número de operações, consumindo energia e tornando o processo pouco competitivo. A hidrólise enzimática apresenta diversas vantagens sobre a química, dentre as quais se devem destacar: menor gasto energético e alta especificidade pelo substrato evitando a produção de compostos indesejáveis. No entanto, a via enzimática é mais lenta e tem como uma das 


\section{9 a 22 de outubro de 2014 \\ Florianópolis/SC}

principais barreiras processos eficientes e viáveis de produção de enzimas a custo que não inviabilize seu uso (Mishima et al., 2006).

A fermentação em estado sólido (FES) é um processo eficiente para obtenção de enzima celulolíticas que podem ser aplicadas na hidrólise de materiais lignocelulósicos. Entretanto, essa técnica ainda carece de conhecimento científico e tecnológico que permita sua aplicação em larga escala para diferentes combinações de microrganismos e substratos, com foco nos fungos filamentosos como agentes fermentativos e nos rejeitos sólidos agroindustriais como substratos econômica e ambientalmente amigáveis. Neste contexto, o objetivo deste trabalho foi avaliar a eficiência do extrato produzido em FES pelo fungo termofílico Myceliophthora thermophila na hidrólise enzimática de bagaço de cana in natura e pré-tratado com ozônio.

\section{MATERIAIS E MÉTODOS}

\subsection{Fermentação em reator de leito empacotado}

A fermentação em reator de leito empacotado foi realizada em sistema similar ao empregado por Umsza-Guez (2009), Zanelato et al. (2012) e Casciatori (2012). O fermentador foi construído em módulos encamisados em aço inox, com 7,62 cm de diâmetro interno e $10 \mathrm{~cm}$ de comprimento, de modo que seu comprimento totalizou $1 \mathrm{~m}$ de altura por meio da adição consecutiva dos 10 módulos.

O cultivo do fungo termofílico Myceliophthora thermophila no biorreator foi realizado em substrato composto por bagaço de cana e farelo de trigo na proporção de 7:3 p/p, com umidade inicial do substrato de $75 \%$, com base nas recomendações de Zanelato et al. (2012). As temperaturas do ar e da camisa foram de $45^{\circ} \mathrm{C}$, temperatura ótima de crescimento e produção de celulases por este fungo, e o tempo de fermentação estipulado foi de 96 horas. A taxa de aeração foi de $240 \mathrm{~L} / \mathrm{h}$.

\subsection{Pré-tratamento do bagaço de cana}

Como matéria prima para a hidrólise enzimática, o bagaço de cana coletado na Usina Vale de Onda Verde - SP foi lavado e seco a $60{ }^{\circ} \mathrm{C}$ até peso constante, triturado e separado em peneira granulométrica de $0,87 \mathrm{~mm}$ para padronização do tamanho da fibra, aproveitando-se a porção que conseguiu atravessar a malha da peneira. O gás ozônio foi obtido usando o gerador Radast 10C Ozoxi-Ozônio e para a irradiação de ultrassom foi empregada sonda ultrassônica operante a $22 \mathrm{kHz}$ e temperatura ambiente.

Para o pré-tratamento do bagaço de cana coletado foram pesados $20 \mathrm{~g}$ de bagaço e umedecidos com $10 \mathrm{~mL}$ de solução de $\mathrm{NaOH} 0,1 \mathrm{~mol} \mathrm{~L}^{-1}$, os quais foram empacotados em coluna de vidro e colocados sob fluxo constante de ozônio durante 1 hora, em seguida o bagaço foi desempacotado e homogeneizado. Após a homogeneização, esse bagaço foi colocado em erlenmeyer, tratado com 400 $\mathrm{mL}$ de solução alcalina $\left(\mathrm{NaOH} 0,1 \mathrm{~mol} \mathrm{~L}^{-1}\right)$ e submetido ao efeito de ultrassom por 5 minutos. Transcorrido o tempo de ultrassom, o bagaço foi mantido em repouso por 2 horas. Por fim, a fração solubilizada correspondente ao pré-hidrolisado foi recolhida, filtrada e utilizada para quantificação de açúcares redutores. $\mathrm{O}$ bagaço tratado foi lavado com água destilada, seco a $60{ }^{\circ} \mathrm{C}$ até obter peso 
constante e armazenado protegido de luz e umidade para posterior utilização na etapa hidrólise enzimática (Perrone, 2012).

\subsection{Hidrólise enzimática}

As hidrólises enzimáticas foram realizadas em erlenmeyer, contendo $0,2 \mathrm{~g}$ de bagaço de cana. Foram adicionados diferentes volumes de extrato enzimático bruto em cada erlenmeyer, de modo a completar o volume final para $10 \mathrm{~mL}$ com tampão ácido acético/ $\mathrm{NaOH} 0,2 \mathrm{M} \mathrm{pH} 5,0$. Os erlenmeyers foram colocados em agitador orbital a $150 \mathrm{rpm}$. As hidrólises foram conduzidas a $65^{\circ} \mathrm{C}$, temperatura ótima de atividade enzimática do extrato produzido pelo fungo Myceliophthora thermophila, já determinada por Casciatori (2012).

Para a etapa de hidrólise enzimática, o planejamento experimental adotado para avaliação da eficiência de sacarificação foi um planejamento de superfície de resposta central composto com 5 repetições do ponto central. Os fatores adotados foram dois, quais sejam volume de extrato enzimático aplicado e tempo de hidrólise, totalizando 13 ensaios para cada extrato enzimático e para cada estado do bagaço de cana (in natura e ozonolisado), sendo 9 tratamentos e 4 testes no ponto central. O fungo empregado neste trabalho e os dois tipos de bagaço de cana foram tratados como blocos do planejamento experimental. A variável de resposta dos ensaios foi a quantidade de açúcares redutores liberados na sacarificação, obtida pelo método de DNS (Miller, 1959).

Tabela 1 - Planejamento experimental central composto dos ensaios de hidrólise enzimática

\begin{tabular}{c|cc|cc}
\hline \multirow{2}{*}{ Ensaio } & \multicolumn{2}{|c|}{ Variáveis codificadas } & \multicolumn{2}{c}{ Variáveis decodificadas } \\
\cline { 2 - 5 } & $\begin{array}{c}\text { Volume de extrato } \\
\text { enzimático (mL) }\end{array}$ & $\begin{array}{c}\text { Tempo de hidrólise } \\
\text { (horas) }\end{array}$ & $\begin{array}{c}\text { Volume de extrato } \\
\text { enzimático (mL) }\end{array}$ & $\begin{array}{c}\text { Tempo de hidrólise } \\
\text { (horas) }\end{array}$ \\
\hline 1 & -1 & -1 & 3 & 2 \\
\hline 2 & 1 & -1 & 7 & 6 \\
\hline 3 & -1 & 1 & 7 & 6 \\
\hline 4 & 1 & 1 & 2,2 & 4 \\
\hline 5 & $-1,41$ & 0 & 7,8 & 4 \\
\hline 6 & 1,41 & 0 & 5 & 6,2 \\
\hline 7 & 0 & $-1,41$ & 5 & 4 \\
\hline 8 & 0 & 1,41 & 5 & 4 \\
\hline 9 & 0 & 0 & 5 & 4 \\
\hline 10 & 0 & 0 & 5 & 4 \\
\hline 11 & 0 & 0 & 5 & 5 \\
\hline 12 & 0 & 0 & 5 & 4 \\
\hline 13 & 0 & 0 & & 5 \\
\hline
\end{tabular}




\section{9 a 22 de outubro de 2014 \\ Florianópolis/SC}

\subsection{Determinação quantitativa de açúcares redutores}

Os açúcares redutores do hidrolisado foram determinados pelo método de Miller (1959). A reação foi conduzida adicionando-se, em tubos, $1 \mathrm{~mL}$ da amostra filtrada, juntamente com $1 \mathrm{~mL}$ do reagente DNS e levados para o banho de ebulição por 10 minutos. Os tubos foram resfriados em banho de gelo por 5 minutos e então, em cada tubo foi adicionado $8 \mathrm{~mL}$ de água destilada. Foi traçada uma curva de calibração utilizando-se glicose como padrão e as amostras foram quantificadas por leitura espectrofotométrica em $540 \mathrm{~nm}$.

\section{RESULTADOS E DISCUSSÕES}

As hidrólises enzimáticas do bagaço de cana foram realizadas de acordo com o planejamento experimental descrito na tabela 1 do item 2.2 dos Materiais e Métodos. Os teores de açúcares redutores totais solubilizados pela hidrólise enzimática do bagaço de cana in natura e pré-tratado com ozônio, determinados pelo método de Miller (1959) e expressos em mg de açúcares redutores por grama de bagaço de cana, são apresentados na Tabela 2.

Os resultados apresentados na Tabela 2 permitem observar que o aumento da quantidade de extrato enzimático resultou em um aumento da liberação dos açúcares, bem como o aumento do tempo de hidrólise ocasionou maior quantificação dos açúcares redutores. Quanto ao tempo de hidrólise, observou-se que o melhor resultado ocorreu com um tempo mais elevado, o que pode estar associado ao fato de que em um tempo menor, provavelmente nem todas as enzimas tinham acabado de catalisar o substrato. Desta forma, o tempo está correlacionado à cinética de catalisação, pois quanto maior o tempo de hidrólise, maior a chance de ocorrer choques entre a enzima e o substrato, sendo limitado pelo tempo reacional em que a hidrólise enzimática é cessada. Contudo, deve-se atentar para o fato de que altos tempos de reação podem provocar a inativação do complexo enzimático. A Tabela 2 também permite observar que para a hidrólise do bagaço de cana pré-tratado com ozônio utilizando-se o extrato enzimático produzido pelo fungo Myceliophthora thermophila por cultivo sólido obteve-se uma maior liberação de açúcares redutores do que para o bagaço de cana in natura. Com esse bagaço, obteve-se uma quantificação máxima de açúcar redutor igual a 147,50 mg de açúcar redutor / g de bagaço tratado empregando-se $7 \mathrm{~mL}$ de extrato enzimático bruto e 6 horas de hidrólise, enquanto para o bagaço de cana in natura a maior quantificação foi igual a $61,82 \mathrm{mg}$ de açúcar redutor / $\mathrm{g}$ de bagaço de cana in natura utilizando-se as mesmas condições de volume e tempo de hidrólise.

Na Figura 1a é possível notar que empregando-se um maior volume de extrato enzimático produzido por FES obteve-se uma maior quantidade de açúcar redutor, pois com volumes menores possivelmente todas as enzimas ficaram saturadas e não sobrou substrato para a hidrólise. Sendo assim, para a faixa de volumes testados quanto maior o volume de extrato enzimático utilizado na hidrólise, maior a eficiência da sacarificação enzimática. No entanto, testes com volumes maiores podem ser realizados até se encontrar o ponto a partir do qual a liberação de açúcares redutores tornese constante. Além disso, a baixa atividade específica do complexo enzimático também está relacionado à exigência de alta relação enzima/substrato para efetivação da hidrólise. Os dados da Figura 1a também permitem observar que não ocorreu a saturação das enzimas pelo substrato, uma 
vez que pela projeção da superfície de resposta ainda deveria ocorrer um aumento crescente na liberação de açúcares redutores em função do tempo de hidrólise e do volume de extrato enzimático empregado na sacarificação do bagaço de cana in natura.

Tabela 2 - Açúcares redutores liberados nas hidrólises enzimáticas do bagaço de cana in natura e pré-tratado com ozônio

\begin{tabular}{|c|c|c|c|c|}
\hline \multirow[b]{2}{*}{ Ensaio } & \multicolumn{2}{|c|}{ Bagaço in natura } & \multicolumn{2}{|c|}{ Bagaço pré-tratado com ozônio } \\
\hline & $\begin{array}{c}\text { Massa de } \\
\text { Bagaço de cana } \\
(\mathrm{g})\end{array}$ & $\begin{array}{l}\text { mg de açúcares } \\
\text { redutores / g de } \\
\text { bagaço }\end{array}$ & $\begin{array}{c}\text { Massa de } \\
\text { Bagaço de cana } \\
(\mathrm{g})\end{array}$ & $\begin{array}{l}\text { mg de açúcares } \\
\text { redutores / g de } \\
\text { bagaço }\end{array}$ \\
\hline 1 & 0,2009 & 29,47 & 0,2010 & 62,19 \\
\hline 2 & 0,2005 & 44,64 & 0,2011 & 94,48 \\
\hline 3 & 0,2002 & 39,66 & 0,2012 & 70,08 \\
\hline 4 & 0,2009 & 61,82 & 0,2000 & 147,50 \\
\hline 5 & 0,2002 & 35,31 & 0,2001 & 35,48 \\
\hline 6 & 0,2009 & 51,17 & 0,2006 & 105,68 \\
\hline 7 & 0,2006 & 38,63 & 0,2007 & 66,27 \\
\hline 8 & 0,2002 & 46,75 & 0,2001 & 66,97 \\
\hline 9 & 0,2011 & 49,73 & 0,2012 & 75,05 \\
\hline 10 & 0,2004 & 41,27 & 0,2006 & 79,26 \\
\hline 11 & 0,2009 & 42,71 & 0,2004 & 68,36 \\
\hline 12 & 0,2002 & 44,86 & 0,2004 & 78,84 \\
\hline 13 & 0,2007 & 41,70 & 0,2010 & 83,58 \\
\hline
\end{tabular}

Similarmente ao bagaço não tratado, o aumento do volume de extrato e do tempo de hidrólise aumentaram a liberação de açúcares redutores para o bagaço ozonolizado conforme é possível observar na Figura 1b. Contudo, em geral, para os ensaios com o bagaço pré-tratado com ozônio notou-se um aumento de aproximadamente $80 \%$ da quantidade de açúcares redutores liberados quando comparado ao bagaço de cana in natura. 


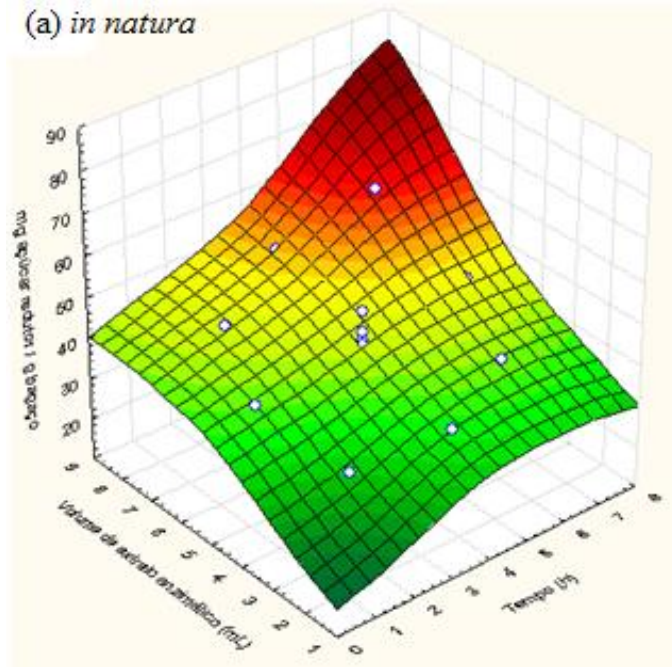

(b) Ozonolisado

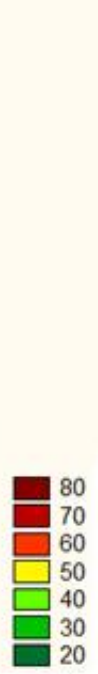

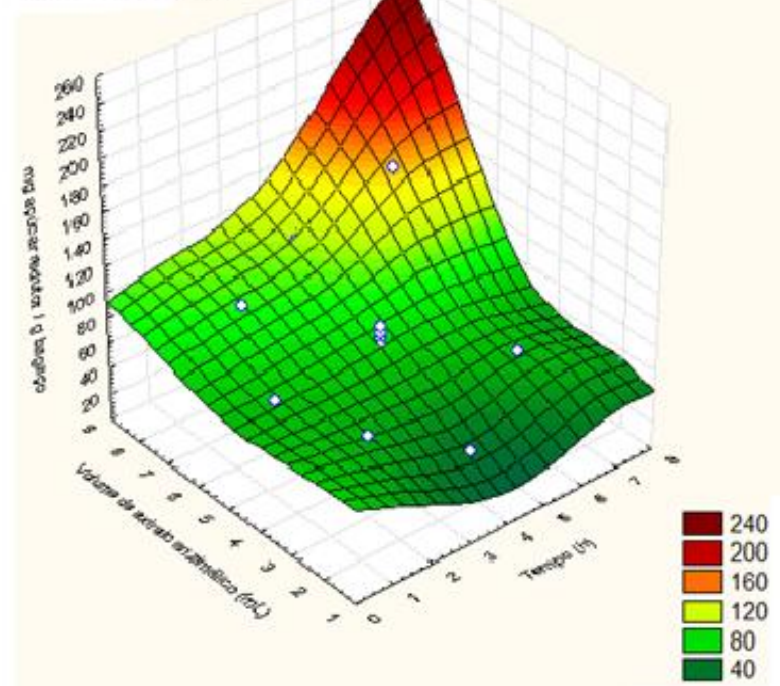

Figura 1 - Superfície de resposta de açúcares redutores em função do tempo de hidrólise e volume de extrato enzimático (a) para o bagaço in natura; (b) para o bagaço pré-tratado com ozônio.

Os estimadores estatísticos dos dados da hidrólise enzimática do bagaço de cana in natura e pré-tratado com ozônio não indicaram diferença significativa na quantidade de açúcares redutores totais liberados com as variações do volume de enzima e do tempo de hidrólise. Contudo, os melhores resultados foram obtidos empregando-se maior volume de enzima e maior tempo de hidrólise como é possível notar na Figura 1.

\section{CONCLUSÃO}

Os melhores resultados de sacarificação de bagaço de cana foram obtidos empregando-se maiores volume de enzima e tempo de hidrólise. Em geral, para os ensaios com o bagaço ozonolizado notou-se um aumento de aproximadamente $80 \%$ da quantidade de açúcares redutores liberados quando comparado ao bagaço de cana in natura. Assim, tem-se que o extrato enzimático produzido pelo fungo Myceliophthora thermophila em reator de leito fixo e o pré-tratamento utilizado no presente trabalho mostraram-se eficientes para aumento da sacarificação enzimática do bagaço de cana.

\section{REFERÊNCIAS}

CASCIATORI, F.P. Biorreatores de leito fixo para fermentação em estado sólido: Ampliação de escala para produção de celulases de origem fúngica. Exame Geral de Qualificação, Universidade Estadual Paulista, São José do Rio Preto, UNESP, 2012. No prelo.

CHANG, V.S.; NAGWANI, M.; HOLTZAPPLE, M.T. Lime pretreatment of crop residues bagasse and wheat straw. Appl. Biochem. Biotechnol., v. 74, p. 135 - 159, 1998. 
GARCÍA-CUBERO, M.T.; GONZÁLES-BENITO, G.; INDACOECHEA, I.; COCA, M.; BOLADO, S. Effect of ozonolysis pretreatment on enzymatic digestibility of wheat and rye straw. Bioresour. Technol., v. 100, p. 1608 - 1613, 2009.

JORGENSEN, H.; KRISTENSEN J.B.; FELBY, C. Enzymatic conversion of lignocelluloses into fermentable sugars: challenges and opportunities. Biofuels, Bioprod. Biorefin., v. 1, p. 119 - 134, 2007.

MILLER, G. L. Use of Dinitrosalicylic Acid Reagent for Determination of Reducing Sugar. Anal. Chem., v. 31, p. 426-428, 1959.

MISHIMA, D.; TATEDA, M.; IKE, M.; FUJITA, M. Comparative study on chemical pretreatments to accelerate enzymatic hydrolysis of aquatic macrophyte biomass used in water purification processes. Bioresour. Technol., v. 97, p. 2166-2172, 2006.

PANDEY, A.; SOCCOL, C. R.; NIGAM, P.; SOCCOL, V. T. Biotechnological potential of agroindustrial residues. I. Sugarcane bagasse, Bioresour. Technol., v. 74, p. 69-80, 2000.

PERRONE, O.M. Tratamentos físico-químicos de bagaço de cana para produção de etanol celulósico por hidrólise enzimática. Trabalho de Conclusão de Curso - Bacharelado em Química Ambiental, Universidade Estadual Paulista, São José do Rio Preto, 2012.

PINTO, T. O. P. Produção de Enzimas Celulolíticas pelos fungos Thermoascus aurantiacus CBMAI 756, Thermomyces lanuginosus, Trichoderma reesei QM9414 e Penicillium viridicatum RFC3 e Aplicação na sacarificação do bagaço de cana de açúcar com diferentes pré-tratamentos. 2010. 91f. Dissertação (Mestrado). Universidade Estadual Paulista, São José do Rio Preto - SP, 2010 .

QUESADA, M.; RUBIO, M.; GOMEZ, D. Ozonation of lignin rich solid fractions from corn stalkes. J. Wood Chem. Technol., v. 19, p. 115 - 137, 1999.

UMSZA-GUEZ, M. A. Produção de poligalacturonase em fermentação em estado sólido pelo fungo Thermomucor indicae-seudaticae N31 em escala de frascos e bioreator de leito fixo. 2009. 106f. Tese (Doutorado), Universidade Estadual Paulista, São José do Rio Preto, 2009.

VIDAL, P.F.; MOLINIER, J. Ozonolysis of lignin - improvement of in vitro digestibility of poplar sawdust. Biomass, v. 16, p. 1 - 17, 1988.

ZANELATO, A.I.; SHIOTA, V.M.; GOMES, E.; THOMÉO, J.C. Endoglucanase production with the newly isolated Myceliophtora sp. i-1d3b in a packed bed solid state fermentor. Braz. J. Microbiol., v. 43, n. 4, p. 1536 - 1544, 2012. 